\title{
Caracterización de LAS ACCIONES PARA LA SEPARACIÓN DE LOS RESIDUOS EN LA FUENTE de los barrios Caracolí, Potosí y Tres EsQuinas de la localidad Ciudad Bolívar
} (Characterization of the actions for the separation of waste in the source of the neighborhoods Caracolí, Potosí and Tres esquinas of the Ciudad Bolivar)

Encuentre este artículo en

http://biblioteca.uniminuto.edu/ojs/index.php/IYD/issue/archive

Para citar este artículo / To cite this article

Santos-Rayo, J - Niño-Sandoval, J - Vargas-Puentes, L. (2017).

Caracterización de las acciones para la separación de los residuos en la fuente de los barrios Caracolí, Potosí y Tres esquinas de la localidad Ciudad Bolívar. Revista Inclusión \& Desarrollo, 4 (I), PAGINAS

\section{Resumen}

Desde hace aproximadamente 30 años las zonas de Caracolí, Potosí y Tres Esquinas han venido generando un cambio muy importante dentro de sus entornos, debido a la difusa línea territorial que hay entre la zona rural y la zona urbana, siendo parte dentro del nuevo plan de ordenamiento territorial, ha traído consigo el aumento de sus habitantes y el poblamiento de las zonas, con personas de escasos recursos económicos, víctimas del conflicto armado y grupos familiares con un deficiente conocimiento en el manejo de los desechos y basuras generadas en los barrios. En concordancia con lo anterior, se genera un inadecuado manejo de los residuos sólidos en las comunidades de Ciudad Bolívar, que ha venido generando una problemática de tipo ambiental y social, causada por el incremento de los residuos sólidos a raíz de la forma en que la población hace el manejo de los mismos, la falta de educación y responsabilidad ambiental desde la separación en la fuente.

Palabas clave: manejo de residuos, caracterización del manejo de residuos, contaminación ambiental, inadecuado manejo de residuos, imaginarios sociales, localidad Ciudad Bolívar.

\section{Abstract}

Approximately 30 years the areas of Caracolí, Potosi and Tres Esquinas have been generating a very important change within their environments, because of the diffuse territorial line that exists between rural and urban areas, being part of the new land use plan, has brought about the increase of its inhabitants and the population of the areas, with individuals of limited economic resources, victims of armed conflict and family groups with a poor knowledge in the management of wastes and wastes generated in the neighborhoods. In concordance with it previous, is generates an inappropriate management of them waste solid in them communities of city Bolivar, that has come generating a problematic of type environmental and social, caused by the increase of them waste solid following it form in that the population makes the management of them same, it lack of education and responsibility environmental from the separation in the source.

Keywords: management of waste, characterization of the handling of waste, air pollution, inadequate waste management, social imaginaries, Ciudad Bolivar locality.

\footnotetext{
'Estudiante de Administración en Salud Ocupacional de la Corporación Universitaria Minuto de Dios UNIMINUTO.

2 Estudiante de Administración en Salud Ocupacional de la Corporación Universitaria Minuto de Dios UNIMINUTO.
}

${ }^{3}$ Magíster en sistemas integrados de gestión de la Universidad de la Rioja (España), Especialista en higiene y salud ocupacional de la Universidad Distrital e Ingeniera industrial de la Universidad Autónoma de Colombia. Docente del programa Administración en Salud Ocupacional de la Corporación Universitaria Minuto de Dios- UNIMINUTO. 


\section{INTRODUCCIÓN}

En Ciudad Bolívar "el medio ambiente es mucho menos que medio". No hay zonas de recreación; el aire está altamente contaminado; los cerros están habitados en su mayoría; las fuentes hídricas son alcantarillas, las calles están completamente cubiertas de basuras y los días de recolección de la misma no son suficientes (Alcaldía local de Ciudad Bolívar, 2013), la cantidad de animales domésticos abandonados y aquellos generadores de enfermedades (roedores) cada día va en aumento. (Alcaldía Mayor de Bogotá, 2016).

Por lo anterior se busca analizar la problemática con el planteamiento de los objetivos de la investigación, caracterizar las acciones para la separación de la fuente de los residuos sólidos de las comunidades, la identificación de los imaginarios definidos como la lógica heredada y el imaginario social (Castoriadis, 1983), los cuales posee la población acerca del concepto residuo-basura y el análisis de las prácticas cotidianas de separación en la fuente realizados por la comunidad; adicional a esto, se identifica la pertinencia de la investigación, basándose bajo tres pilares, el primero de ellos está en la formación orientada en el desarrollo de conocimientos que sean aplicables al beneficio de la comunidad, la investigación para la identificación de las problemáticas de las comunidades y brindar soluciones para las mismas y la praxeología debido a que se quiso estudiar la estructura lógica de la acción humana en cuanto a las características y los imaginarios que vienen con ellos para la separación en la fuente de los residuos.

El siguiente enfoque estuvo dado en el ámbito educativo; el ser perteneciente a una comunidad académica

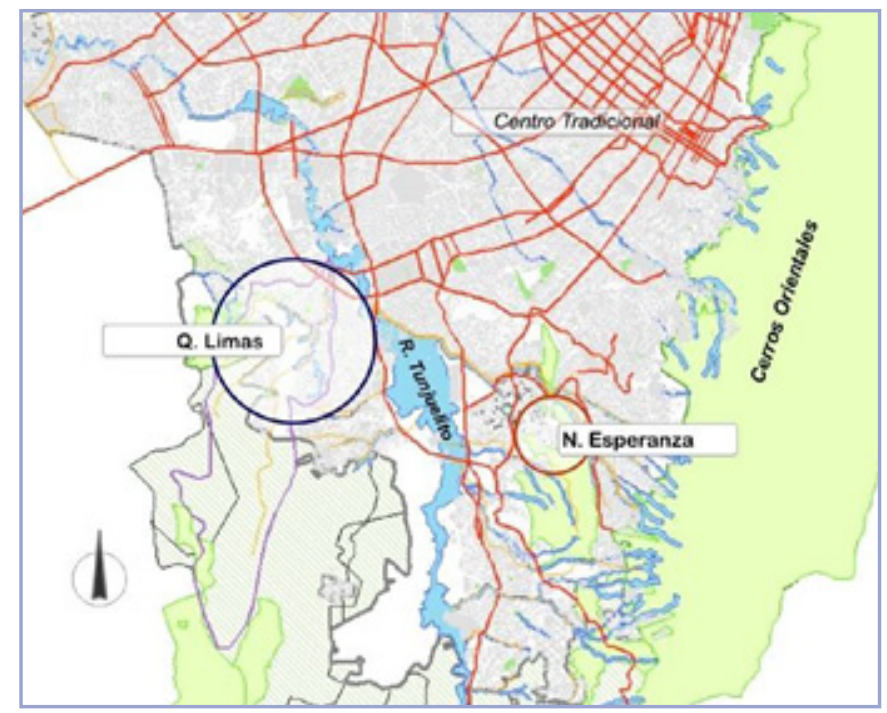

Figura I. Localización general Sectores objeto del Plan. Fuente: Grupo consultor. con alto sentido de responsabilidad social, y su enfoque en la generación de profesionales comprometidos con la ayuda a las comunidades en busca de contribuir a mejorar la calidad de vida y por último un ámbito cultural, teniendo en cuenta que las investigaciones de tipo cualitativo dirigidas a poblaciones están sujetas siempre a la herencia y sus imaginarios culturales, además de aclarar que los habitantes de estos territorios en una gran proporción son pertenecientes o traen consigo concepciones de grupos étnicos característicos los cuales se reconocen a partir de sus costumbres y tradiciones que permiten construir un sentido de pertenencia con la comunidad de origen (Ministerio de Educación Nacional, 2016), generando de esta manera una población productiva en los territorios pero expuesta en mayor proporción a los determinantes sociales de la localidad como lo son la delincuencia, la inseguridad entre otros. Para el año 2016, esta población ha venido en aumento debido a la poca accesibilidad a los servicios de salud y seguridad social y el esparcimiento por el territorio el cual ha sido invadido por diferentes grupos de personas.

\section{Planteamiento DEL PROBLEMA}

Los residuos existen desde que nuestro planeta tiene seres vivos, hace unos 4.000 millones de años. Antiguamente, la eliminación de los residuos humanos no planteaba un problema significativo, ya que la población era pequeña y la cantidad de terreno disponible para la asimilación de los residuos era grande. Sin embargo, la problemática de los residuos comienza con el desarrollo de la sociedad moderna en la que se habita, no sólo en el aspecto referido a la cantidad de residuos que ésta genera (difíilmente asimilable por la naturaleza), de manera importantísima, a la calidad de los mismos (Garri-

(1)

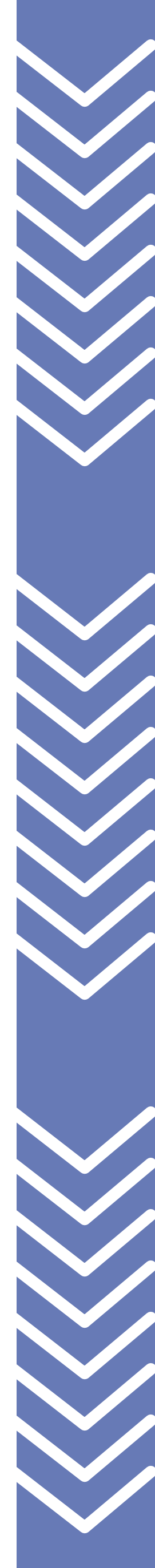


gues, 2003). Este problema de la gestión de los residuos existe y se agrava año tras año. Ante tal situación, resulta importante analizar los factores que han incrementado de manera tan alarmante el problema de los residuos urbanos. En general, pueden señalarse cuatro causas principales:

I. El rápido crecimiento demográfico.

2. La concentración de la población en los centros urbanos.

3. La utilización de bienes materiales de rápido deterioro.

4. El uso cada vez más generalizado de envases sin retorno, fabricados con materiales no degradables (MOPT, 1992).

La gestión incorrecta de los residuos sólidos urbanos genera entre otros, los siguientes problemas (FICYT, 1998):

- La presencia de residuos abandonados produce una sensación de suciedad a la vez que deterioran el paisaje.

- Los depósitos incontrolados de residuos sólidos urbanos producen, al fermentar, olores muy molestos.

- Los residuos fermentables son fácilmente autoinflamables por lo que pueden provocar incendios que ocasionan una contaminación atmosférica muy desagradable para la vecindad y, en ocasiones, peligrosa para la circulación y para la seguridad de los bosques cercanos.

- Un vertido de residuos realizado sin ningún tipo de control, presenta un grave riesgo de contaminación de las aguas tanto superficiales como subterráneas, con el consiguiente peligro para la salud si son utilizadas para el abastecimiento de agua potable a la población.

- Los residuos orgánicos favorecen la existencia de gran cantidad de roedores e insectos que son agentes portadores de enfermedades y algunas contaminaciones bacterianas.

En las distintas sociedades el manejo de los desechos, son tratados de manera indiscriminada, ocasionado con este comportamiento, grandes daños ambientales y pérdidas económicas que las afectan. En primera instancia porque gran parte de los desechos que se generan diariamente a las estaciones de basura, tienen un valor económico, estos residuos son susceptibles de reutilización o reciclaje. En segundo lugar, al botarlos o tirarlos en cualquier sitio se impacta negativamente el ambiente y se deteriora la salud pública.

Teniendo en cuenta lo anteriormente mencionado, se toma como zonas de estudio, los barrios Caracolí, Potosí, y Tres Esquinas se encuentran ubicados en la localidad de Ciudad Bolívar en la UPZ 70. Los límites se definen por el norte con los barrios Brisas y Jerusalén, al sur se conecta con los barrios Cazucá y Ciudadela sucre (Municipio de Soacha), al oriente se encuentra el sector la Isla y al occidente con Sierra Morena. Las zonas cuentan con espacios muy reducidos de material vegetal, perteneciendo en un gran porcentaje a las quebradas (Limas) que se encuentran altamente contaminadas por

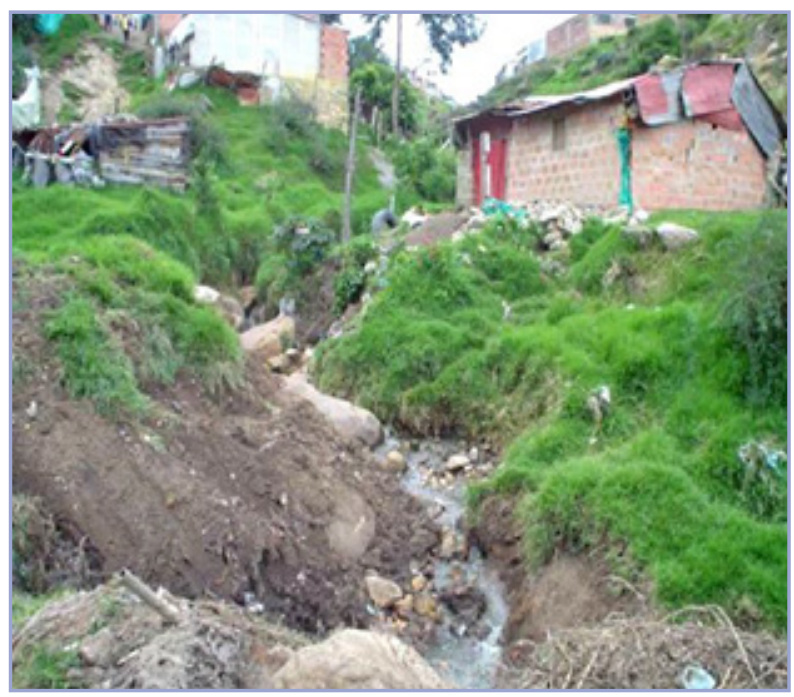

Fotografía No. I. Viviendas en Zonas de Riesgo Quebrada Limas. Fuente: DPAE. 
intervención antrópica (Alcaldía local de Ciudad Bolívar, 20I3).

La quebrada Limas con 10.5 kilómetros de longitud recorre gran parte de la localidad de Ciudad Bolívar y desemboca por la parte izquierda del río Tunjuelo. Desde 1994, se reporta la contaminación, en la parte media y baja de la quebrada.

En la quebrada Limas existe una contaminación por arrojo de basuras y escombros. También esta fuente se afectada por los pasos peatonales y vehiculares que generan obstrucción del cauce, incluida la construcción de viviendas sobre la misma. Así mismo esta fuente hídrica es utilizada como un desecho de residuos sólidos.

El tipo de problemáticas ambientales que acogen a la población, desde los niños hasta las personas más longevas, se plantea realizar la siguiente investigación orientada a identificar la caracterización de las acciones para la separación de los residuos sólidos en la fuente que son generados por los barrios Caracolí, Potosí y Tres esquinas, buscando minimizar los riesgos de adquirir enfermedades infecto contagiosas, la contaminación visual, los riesgos en salud pública, y permitir de esta manera que las comunidades se apropien y adquieran sentido de pertenencia con los territorios para que ellos sean los principales actores con la participación continua para la disminución y control de la contaminación ambiental de la localidad.

La disposición inadecuada de los residuos sólidos generados por las comunidades de los barrios Caracolí, Potosí, y Tres Esquinas, permite identificar la inocultable contaminación en los territorios, incluyendo un inminente riesgo en la salud pública tal como lo asegura Juan Guillermo Rubio, (médico veterinario centro de acogida de la alcaldía) quien menciona que se puede generar problemas relacionado con la presencia de enfermedades Zoonóticas como lo son el aumento de los animales domésticos abandonados y aquellos clasificados como vectores (insectos y roedores) causantes enfermedades como la leptospirosis, teniasis, y entre las más comunes la toxoplasmosis, de las cuales una son causadas por la presencia de animales sin control en su crecimiento poblacional. (Alcaldía Mayor de Bogotá, 2016).

Desde los diagnósticos observacionales, se evidencia un escaso conocimiento de la problemática (Instituto de estudios ambientales IDEA, Universidad Nacional, 1994) de contaminación ambiental que aqueja a las poblaciones, la baja apreciación de los territorios en los cuales habitan las personas, permitieron claramente orientar la investigación realizada, dando prioridad a el conocimien- to y el análisis de las percepciones que tiene consigo la comunidad de los barrios acerca del significado en cuanto a la separación de desechos sólidos para así generar pautas para la investigación y establecer los resultados.

\section{Metodología}

A partir de todo esto, se decide realizar la investigación desde un enfoque cualitativo, teniendo en cuenta que se busca la descripción de los eventos o fenómenos de estudio, a partir de las experiencias vividas y los datos observados, además se incluyó el observar las situaciones desde una visión holística - integrativa, siendo considerado el fenómeno de estudio como un todo, estudiando antecedentes, concepciones socioculturales, factores influyentes e interacciones con el entorno.

Se tuvo en cuenta referentes de investigación como Ernesto Rojas Morales y entidades como el Hospital Vista Hermosa I nivel ESE, la alcaldía mayor de Bogotá, el ministerio de educación entre otros, quienes han venido realizando estudios y observaciones acerca de la problemática en cuestión; todo esto bajo el diseño metodológico descriptivo-interpretativo (Aguirre 1997).

Después de esto, se instauraron tres fases para la ejecución de la investigación, se partió de una primera fase, donde se realizó la recolección de datos mediante entrevistas semiestructuradas a líderes comunitarios, habitantes de las comunidades, establecimientos comerciales y a las personas quienes viven del reciclaje de los residuos sólidos; consecutivo a esto se instauró la segunda fase, realizando el análisis de la información y los datos recolectados a partir de los recorridos observacionales, las revisiones bibliográficas, el análisis de los documentos, los resultados obtenidos de las entrevistas y la elaboración de informes para ser entregados a los tutores del semillero de investigación, sujetos correcciones y modificaciones bajo la mirada de la investigación científica.

En una última instancia, se inició con una tercera fase de la investigación donde se realizó la presentación de los resultados en ponencias de los semilleros de investigación del área de administración en salud ocupacional por parte de la Corporación Universitaria Minuto de Dios en sus diferentes sedes, dando a conocer todos los resultados obtenidos mediante la elaboración de un póster (figura 2) realizados por cada uno de los grupos ponentes, allí se recibieron las observaciones pertinentes por un panel de expertos en la investigación científica 
y la metodología de la investigación, realizando las respectivas correcciones para las siguientes presentaciones.

\section{RESULTADOS}

Partiendo de los instrumentos aplicados para la recolección de datos como lo fueron las entrevistas semiestructuradas se establecen tres categorías de clasificación de los resultados entre las cuales se evidencian, la concepción de residuo-basura en relación con el lugar de agrupación de los mismos; la disposición de los desechos sólidos relacionados con las fuentes hídricas y los imaginarios de las comunidades respecto a la reutilización de los residuos.

Bajo los recorridos observacionales, se identificó que, disposición inadecuada de residuos se produce cuando la comunidad transforma en botaderos de basu- ra las calles principales, los parques, los lotes vacíos y las fuentes de agua.

La concepción de residuo-basura en relación con el lugar de disposición de los residuos se identifica que el conocimiento que algunas personas de las comunidades tienen acerca del manejo de los desechos, es una mínima clasificación de residuos y una superficial identificación de aquellos que pueden ser reutilizados; mencionando que son pocas las familias o establecimientos comerciales que poseen este tipo de conocimiento, también, se evidenció la gran cantidad de basuras en las calles de los sectores donde la mayor proporción es generada por hogares; la inadecuada disposición de los residuos genera que se incremente la cantidad de basuras en las calles a raíz de no poseer un lugar específico para el desecho de los mismos, incluida la no separación de los residuos desde los hogares.

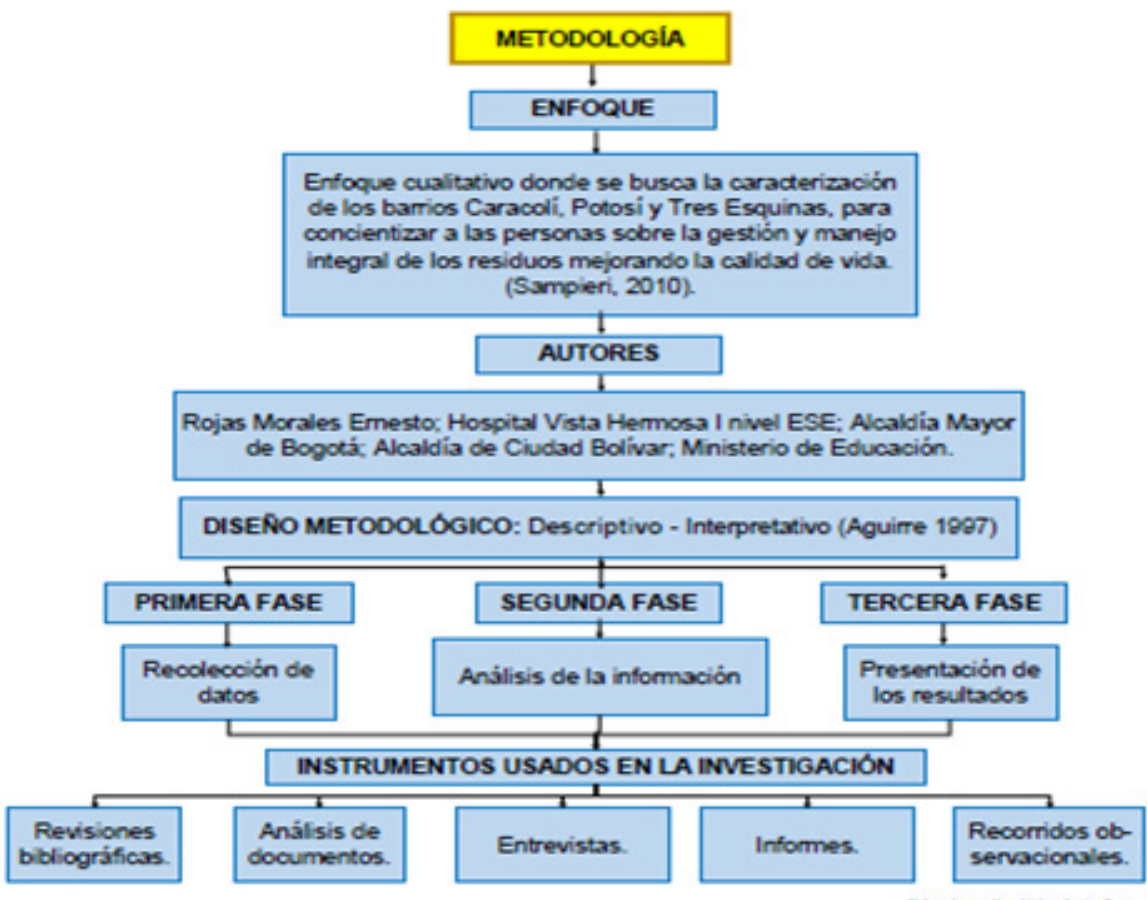

\section{RESULTADOS}
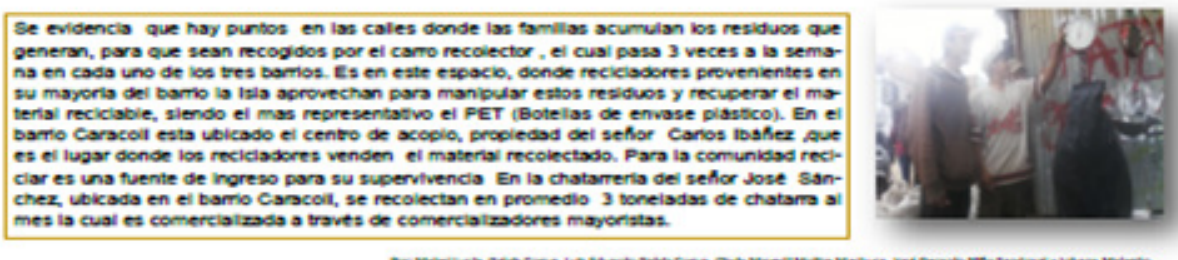

Figura 2. Poster investigación "Caracterización de las acciones para la separación de los residuos en la fuente en los barrios Caracolí, Potosí y Tres Esquinas". 
En cuanto a los líderes comunitarios se identificó que han existido una serie de intervenciones por parte de entidades gubernamentales como lo son Aseo Capital y el Hospital Vista Hermosa, quienes han desarrollado diferentes proyectos incentivando a la población en cuanto a la separación de residuos desde los hogares, siendo estos la fuente principal generadora de los residuos sólidos. (Hospital Vista Hermosa I Nivel, 20I0).

A partir de esto y durante los recorridos observacionales realizados en los barrios Caracolí, Potosí y Tres Esquinas, el posterior análisis de resultados obtenidos, cabe mencionar que estos proyectos no han llegado a culminar por la falta de seguimiento por parte de las entidades, interés por parte de la comunidad y la mínima capacitación hacia los hogares, comerciantes, colegios y personas dedicadas al reciclaje de los residuos, identificando que carecen de conocimientos frente al tema.

Ahora bien, FICYT indica que las fuentes hídricas se han venido convirtiendo en el vertido de residuos sin ningún tipo de control, presentando un grave riesgo de contaminación de las aguas tanto superficiales como subterráneas, con el consiguiente peligro para la salud si son utilizadas para el abastecimiento de agua potable a la población. La disposición de los desechos sólidos en relación con las fuentes hídricas, se logra identificar que las comunidades desechan los residuos en las fuentes hídricas por distintas razones, una de estas radica en la ubicación territorial de los hogares en relación a las fuentes hídricas y la gran distancia a los centros de acopio, son un factor clave que contribuye a que los habitantes de las comunidades desechen los residuos allí, convirtiendo las pequeñas quebradas en receptores de los desechos, conocidos como caños (FICYT, 1998).

Los imaginarios que las comunidades poseen respecto a la reutilización de los residuos, permite identificar durante las entrevistas semiestructuradas realizadas a personas que laboran en el reciclaje de las zonas, la existencia de puntos de recolección ubicados generalmente en las esquinas de las calles, en donde un gran porcentaje de la población acumulan los residuos sólidos generados, y estos son recogidos por los carros recolectores de la basura dispuestos por el Distrito tres veces por semana, es aquí donde las personas recicladoras antes de que pase el vehículo recolector de la basura, se tomen el tiempo de clasificar y tomar el material sólido reciclable para poder venderlo. Luego los residuos reciclables son llevados establecimientos de acopio donde pueden generar un positivo provecho de los residuos sólidos, utilizados por ellos sin ninguna precaución de no destruir los empaques donde son dispuestos los residuos o simplemente dejar todo en un debido orden; junto con lo anterior, en las respuestas generadas por los líderes comunitarios, comerciantes y a los habitantes de las comunidades se logra identificar una desatención por parte de la comunidad respecto a la problemática ambiental de la cual todos son partícipes.

Adicionalmente el imaginario que más predomina en las comunidades es que todo lo generado o utilizado por los habitantes que ya no sirve es basura, clasificándolo como un problema sociocultural que se debe de corregir.

\section{CONCLUSIONES}

A partir de los resultados obtenidos durante la investigación, se logra la caracterización de las acciones para la separación de los residuos en tres comunidades específicas de la localidad de Ciudad Bolívar, concluyendo que evidentemente la contaminación en estos territorios es de gran impacto, afectando en una gran proporción a los habitantes de estos sectores, en cuanto a su estado de salud, su economía y su entorno ambiental.

Se identificó la influencia que tiene los imaginarios culturales en la comunidad acerca del manejo los desechos y su "reutilización", con la premisa de "todo lo generado o utilizado por los habitantes que ya no sirve es basura", enmarca las conductas para el manejo de los residuos, teniendo en cuenta la poca información acerca de la problemática por parte de la comunidad, incluido el bajo interés territorial por parte de la misma.

Se evidencia que las conductas de las personas que habitan cada uno de los territorios del estudio, están relacionadas a las concepciones culturales de cada uno y las prácticas adquiridas en el diario vivir, el indiscriminado desecho de los residuos en cualquier lugar es punto clave que orientó la investigación, dando punto de partida para la búsqueda de posibles soluciones basadas en las conductas que tienen las personas para el manejo de los residuos.

OA partir de todo lo anteriormente mencionado, se identifica que es necesaria la intervención urgente e inmediata en estas comunidades para la mitigar el impacto social y ambiental que ha venido generando la contaminación causada por el inadecuado manejo de los residuos, provocando de esta manera cambios significativos en el estado de salud de la población, disminución de la calidad de vida de la misma y alteraciones en los ciclos ambientales. Los cambios radican en intervenir en las acciones para el manejo de los residuos por parte de las personas en reorientación y mitigación los impactos sociales y ambientales de la problemática actual $\mathbb{2}$ 


\section{Referencias bibliográficas}

Aguirre, A. (1997). Etnografía. En A. Aguirre (Ed.), Etnografía (Metodología cualitativa de la investigación sociocultural) (pp. 3-20). México: Alfaomega Marcombo.

Alcaldía Mayor de Bogotá, DPE, DAPD. (MARZO de 2005). Jimmy. f. h universidad del Tolima lbagué, Colombia.

Alcaldía Mayor de Bogotá; Ley 1259 de 2008; Recuperado de [http://www.alcaldiabogota.gov.co/sisjur/normas/Normal .jsp?i=34388]

Alcaldía local de Ciudad bolívar. (2013). Conociendo mi localidad. Diciembre 03, 20I3, de Alcaldía Mayor, Recuperado de: http://www. ciudadbolivar.gov.co/index.php/descargas/category/I03-ubicacion\#page

Alcaldía Mayor de Bogotá. (s.f.). Recuperado el 4 de Marzo de 2016

Alcaldía Mayor de Bogotá, DPE, DAPD. (MARZO de 2005). Recuperado el 4 de Marzo de 2016

Alcaldía Mayor de Bogotá, (2016); Recolección canina, un aporte a la salud pública de Bogotá; Recuperado de: http://www.bogota.gov.co/ article/temas-de-ciudad/salud/recoleccion-canina-un-aporte-a-la-salud-publica-de-bogota

Angarita Lozano, Diana Liseth (2010); El aprovechamiento de residuos sólidos y su incidencia cultural, social y ambiental en Tunja; Universidad Santo Tomas - Tunja, Vol. I. Núm. I; Recuperado de: http://revistas.ustatunja.edu.co/index.php/ingeniomagno/article/ view/I5

Banco Mundial BIRF - AIF (2016); Basura cero - Los residuos sólidos en el epicentro del Desarrollo Sostenible; marzo 3 de 2016; Recuperado de: http://www.bancomundial.org/es/news/feature/2016/03/03/waste-not-want-not---solid-waste-at-the-heart-ofsustainable-development

Banco Mundial BIRF - AIF (20I2); Argentina: mejores soluciones para el reciclaje y el manejo de restos sólidos; octubre 29 de 2012 ; Recuperado de: http://www.bancomundial.org/es/news/feature/2012/10/26/argentina-urban-solid-waste-management-best-practices

Calendarios y geografías. (2015). Categorías Análisis entorno geográfico. Julio 3I, 20I5, de YouTube Recuperado de: https://www. youtube. $\mathrm{com} /$ watch?v $=\mathrm{uXpGmRBv3IE}$

Castoriadis, Cornelius, 1983; La institución imaginaria de la sociedad, vol. Barcelona: Tusquets Editores.

Costa, F; García, C; Hernández, T. y Polo, A. (1995). Residuos orgánicos urbanos. Manejo y utilización. Consejo Superior de Investigaciones Científicas. Centro de Edafología y Biología Aplicada de Segura. Murcia. I8I pp.

Corporación Taliber, « Potosí-La Isla. Historia de una lucha », Bogotá, 1998. Recuperado Marzo 3 de 2016.

Correa Liliana Constanza (2003); Proyecto de educación ambiental y propuesta de un proyecto piloto de reciclaje en el barrio San Luis Colmena III. Ciudad Bolívar; Pontificia Universidad Javeriana; mayo de 2003; Recuperado de: http://oab2.ambientebogota.gov.co/ es/documentacion-e-investigaciones/resultado-busqueda/proyecto-de-educacion-ambiental-y-propuesta-de-un-proyecto-piloto-dereciclaje-en-el-barrio-san-luis-colmena-iii-ciudad

Entrevista personal a Mauricio Sanabria, Bogotá, I de noviembre de 2007.Recuperado Marzo tres de 2016.

Ernesto Rojas Morales. (2007). Colombia, Una Nación Multicultural; Su diversidad étnica. II de marzo de 2016, de DANE (Departamento Administrativo Nacional de Estadística) Recuperado de: http://www.dane.gov.co/files/censo2005/etnia/sys/colombia_ nacion.pdf

GARRIGUES (2003). Manual para la Gestión de los Residuos Urbanos. El consultor de los ayuntamientos y de los juzgados. Ecoiuris. Madrid. 909 pp.

González Terreros, María Isabel “Escuela-comunidad. Historia de la organización comunitaria en Potosí-Jerusalén, Desde Abajo, Bogotá, 2004. Recuperado marzo tres de 2016.

FICYT (Fundación para el Fomento en Asturias de la Investigación Científica Aplicada y la Tecnológica) (1998). Contaminación e Ingeniería Ambiental. Degradación del suelo y tratamiento de residuos. Oviedo. 436 pp.

Hernández Sampieri, R., Fernández Collado, C., \& amp; Baptista Lucio, P. (2010). Metodología de la investigación, Quinta edición. Bogotá: Mc Graw Hill.

Hontoria García, E. y Zamorano Toro, M. (2000). Fundamentos del manejo de los residuos urbanos. Colección sénior 24. Colegio de Ingenieros de Caminos, Canales y Puertos. España. Pp. 756. 
Caracterización de las acciones para la separación de los residuos en la fuente de los barrios Caracolí, Potosí y Tres Esquinas de la localidad Ciudad Bolívar

Hospital Vista Hermosa I Nivel ESE. (2010). Análisis de la situación de salud, Localidad Cuidad Bolívar. 2016, de Secretaria Distrital de Salud. Recuperado de: http:// www.saludcapital.gov.co/sitios/VigilanciaSaludPublica/Todo\%20ASIS/CIUDAD\%20BOLIVAR.pdf

Instituto de Estudios Ambientales IDEA, 1994; Cúmulo de contaminación en Ciudad Bolívar; Recuperado de: http://www.eltiempo.com/ archivo/documento/MAM-80668

Jaramillo Jorge (2002); Efectos de la inadecuada gestión de residuos sólidos; Universidad de Antioquia, Centro Panamericano de Ingeniería Sanitaria y Ciencias del Ambiente, 2002; Recuperado de: http://www.ecociudadsa.com/verArticulo.php?contenidolD=I I

Leiva Bautista Claudia Cecilia, MSc (2000); Estudio de la opinión de los estudiantes de la Universidad Francisco Gavidia sobre la separación y reciclaje de la basura; Theorethikos: la revista electrónica de la UFG, año III, No. 4; octubre - diciembre 2000; Recuperado de: http://www.ufg.edu.sv/ufg/theorethikos/octubre20/cientifico04.htm

Medina Bermúdez Clara Inés, Ph. D. (1999); Manejo de Residuos Sólidos; Revista de la facultad de ingeniería, Universidad Militar Nueva Granada, noviembre de 1999; Recuperado de: file://C:/Users/mairon/Downloads/Manejo\%20de\%20residuos\%20solidos\%20 UMNG.pdf

Ministerio de Educación Nacional, República de Colombia. (2016). Grupos Étnicos. II de marzo de 2016, de Ministerio de Educación Nacional. Recuperado de: http:// www.colombiaaprende.edu.co/html/mediateca//607/article-84457.html

MOPT (Ministerio de Obras Públicas y Transporte) (1992). Atlas Nacional de España. Edafología. Sección Il. Grupo 7. Ed. Centro Nacional de Información Geográfica. Madrid.

Muerza Fernández Alex (2016); Las ciudades con la mejor gestión de residuos del mundo; enero I3 de 20I6; EROSKI CONSUMER; Recuperado deb: http://www.consumer.es/web/es/medio_ambiente/urbano/2016/01/13/223208.php

Roca Fernández Ana Isabel (2010); Problemática, clasificación y gestión de los residuos sólidos urbanos; AGRI - NOVA Science, Centro de investigaciones agrarias de Mabegondo. Recuperado de: http://www.infoagro.com/documentos/problematica_clasificacion_y_ gestion_residuos_solidos_urbanos.asp

Rojas Morales, Ernesto (2007). Colombia, Una Nación Multicultural; Su diversidad étnica. I I de marzo de 2016, de DANE (Departamento Administrativo Nacional de Estadística) Recuperado de: http://www.dane.gov.co/files/censo2005/etnia/sys/colombia nacion.pdf

Sáez Alejandra, Urdaneta Joheni (2014); Manejo de residuos sólidos en América Latina y el Caribe; Revista Omnia No.3; Universidad de Zulia; pp. I2I-I35; Recuperado de: http://www.redalyc.org/html/737/73737091009/

TCHOBANOGLOUS, G.; THEISEN, H. y VIGIL, S. A. (1994). Gestión integral de residuos sólidos. Ed. Mc Graw-Hill. Madrid. II 07 pp.

Tobasura Isaías (2007); Ambientalismos y ambientalistas: una expresión del ambientalismo en Colombia en Ambiente y sociedad, Campinas, Vol. X, No. 2, 2007, pp. 46. 